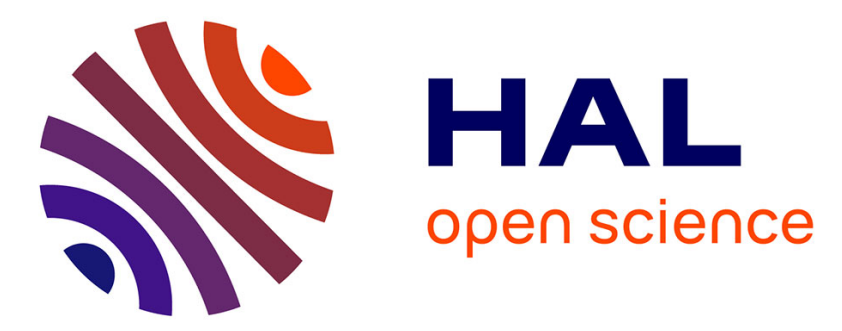

\title{
In situ phase transition of microemulsions for parenteral injection yielding lyotropic liquid crystalline carriers of the antitumor drug bufalin
}

Yawen Li, Angelina Angelova, Jianwen Liu, Vasil M Garamus, Na Li, Markus Drechsler, Yabin Gong, Aihua Zou

\section{To cite this version:}

Yawen Li, Angelina Angelova, Jianwen Liu, Vasil M Garamus, Na Li, et al.. In situ phase transition of microemulsions for parenteral injection yielding lyotropic liquid crystalline carriers of the antitumor drug bufalin. Colloids and Surfaces B: Biointerfaces, 2019, 173, pp.217-225. 10.1016/j.colsurfb.2018.09.023 . hal-02381456

\section{HAL Id: hal-02381456 https://hal.science/hal-02381456}

Submitted on 26 Nov 2019

HAL is a multi-disciplinary open access archive for the deposit and dissemination of scientific research documents, whether they are published or not. The documents may come from teaching and research institutions in France or abroad, or from public or private research centers.
L'archive ouverte pluridisciplinaire HAL, est destinée au dépôt et à la diffusion de documents scientifiques de niveau recherche, publiés ou non, émanant des établissements d'enseignement et de recherche français ou étrangers, des laboratoires publics ou privés. 


\title{
In situ phase transition of microemulsions for parenteral injection yielding lyotropic liquid crystalline carriers of the antitumor drug bufalin
}

\author{
Yawen $\mathrm{Li}^{\mathrm{a}}$, Angelina Angelova ${ }^{\mathrm{b}}$, Jianwen Liu ${ }^{\mathrm{a}}$, Vasil M. Garamus ${ }^{\mathrm{c}}$, \\ $\mathrm{Na} \mathrm{Li}^{\mathrm{d}}$, Markus Drechsler ${ }^{\mathrm{e}}$, Yabin Gong ${ }^{\mathrm{f}}$, Aihua Zou ${ }^{\mathrm{a} *}$ \\ ${ }^{a}$ Shanghai Key Laboratory of Functional Materials Chemistry, State Key Laboratory \\ of Bioreactor Engineering and Institute of Applied Chemistry, School of Chemistry \\ and Molecular Engineering, East China University of Science and Technology, \\ Shanghai 200237, P. R. China \\ ${ }^{b}$ Institut Galien Paris-Sud, CNRS UMR 8612, Univ. Paris-Sud, Université \\ Paris-Saclay, LabEx LERMIT, F-92296 Châtenay-Malabry cedex, France, \\ ${ }^{c}$ Helmholtz-Zentrum Geesthacht, Centre for Materials and Coastal Research, \\ D-21502 Geesthacht, Germany \\ ${ }^{d}$ National Center for Protein Science Shanghai and Shanghai Institute of \\ Biochemistry and Cell Biology, Shanghai 200237, P. R. China \\ ${ }^{e}$ Keylab "Electron and Optical Microscopy", Bavarian Polymerinstitute (BPI), \\ University of Bayreuth, D-95440 Bayreuth, Germany \\ ${ }^{f}$ Yueyang Hospital of Integrated Traditional Chinese and Western Medicine, Shanghai \\ 200437, P.R. China
}

\footnotetext{
"To whom correspondence should be addressed.

Tel/Fax: +86-21-64252231

E-mail: aihuazou@ecust.edu.cn

${ }^{\dagger}$ East China University of Science and Technology.
} 


\section{Abstract}

In this work, we use the small angle X-ray scattering (SAXS) method for controlled preparation of in situ forming sustained-release carriers of the antitumor drug bufalin (BUF), which has very poor solubility and a considerable cardiotoxicity in a non-encapsulated state. To that aim, we exploit the pseudo-ternary phase diagram of an oil(O)/surfactant(S)/water(W) system containing medium chain capric/caprylic triglycerides (MCT) and a co-surfactant blend of Macrogol (15)-hydroxystearate (Solutol HS 15) and sorbitan monooleate (Span 80). Two compositions with different oil contents (B-LC-ME and C-LC-ME) are selected from the microemulsion region of the diagram in order to study the effect of the aqueous environment on their structural behaviour. A phase transition from a microemulsion to a lamellar liquid crystalline phase and multilamellar vesicles is established by SAXS upon progressive dilution. The drug bufalin (BUF) is encapsulated in the microemulsions, which have low viscosity, whereas the release of the drug occurred from the in situ generated lamellar liquid crystalline structures. The formulations are characterized by SAXS, dynamic light scattering (DLS), cryogenic transmission electron microscopy (Cryo-TEM), rheology, drug loading and entrapment efficiency, and in vitro release profiles. A correlation is suggested between the structures of the in situ phase-transition formed LC-ME formulations and the differences in their viscosities and drug release profiles. The performed cytotoxicity, cell apoptosis and pharmacokinetic experiments show an enhanced bioavailability of BUF after encapsulation. These results suggest potential clinical applications for the obtained safe in situ phase-transition sustained-release formulations of BUF.

Keywords: bufalin encapsulation, in situ phase transition, liquid crystalline carrier, SAXS, drug release, bioavailability 


\section{Introduction}

Lipid- and oil-based drug delivery systems are widely used for increasing the solubility and bioavailability of hydrophobic drugs [1-5]. Various types of nanostructured lipid carriers, liquid crystalline lipid nanoparticles (cubosomes, spongosomes, hexosomes, liposomes), microemulsions, nanoemulsions, nanogels, solid lipid nanoparticles, and hybrid lipid-polymer nanoparticles have been investigated with the aim of encapsulation and protection of poor soluble or instable therapeutic molecules [6-15]. The challenges in the development of such formulations comprise (i) reduced therapeutic effects owing to rapid drug release from the carriers, (ii) drug precipitation at the injection site in the cases when the administered quantity exceeds the solubility limit of the hydrophobic drug, and (iii) side effects associated with toxicity or immunoreactions [12-15]. Parenteral administration is performed by injections, which can be intramuscular, subcutaneous, intralesional, intravitreal, and intra-articular among other possible injection sites [16]. So the parenteral routes of drug administration are suitable for treatment of patients using a limited number of injections [16]. Therefore, they require new strategies for facile fabrication of sustained release delivery systems.

In situ phase transitions have emerged as a promising strategy for sustained-release drug delivery applications based on intramuscular, subcutaneous, intra-articular, and intratumoral injections [17-23]. For instance, the phase transition from microemulsion (ME) to liquid crystalline (LC) structures appears as an alternative to the injectable in situ forming polymeric gels [20,24,25] that may provide sustained release profiles of encapsulated drugs. In this approach, the drug encapsulation is made in the microemulsion domain of the pseudo-ternary phase diagram of appropriate oil/surfactant/water systems, where the formulations have low viscosity. Microemulsions (ME) have been extensively studied as drug delivery vehicles for various administration routes [9,10, 12-15]. They have a capacity of solublizing either lipophilic or hydrophilic drug compounds. The progressive dilution 
of the microemulsion by water (upon its contact with the aqueous environment in the body) may result in the formation of a liquid crystalline (LC) carrier of increased viscosity. The latter may favor the diminishment of the drug diffusion rate and provide prolonged drug release [17].

The possibility to exploit in situ phase transitions of drug carrier systems for tuning of their physico-chemical properties and drug release kinetics is expected to improve the therapeutic outcome of several poor soluble drugs, and in particular, to overcome the failure of some of the previously studied delivery systems of bufalin (BUF).

Bufalin (Fig. 1a) is an antitumor compound isolated from the traditional Chinese medicine "Chan'su" (toad venom). It is a bufanolide steroid compound. The broad spectrum of anticancer activities of BUF has received considerable attention [26-32]. The mechanisms of bufalin-induced cell death comprise its (i) influence on the expression of apoptosis-related genes, (ii) inhibition of tumor cell proliferation, (iii) generation of reactive oxygen species, (iv) endoplasmic reticulum stress, (v) JNK (c-Jun N-terminal kinase) activation, (vi) protein kinase modulating effect, (vii) caspase- and mitochondria-dependent signaling pathways, and (viii) cell cycle arrest via the c-Myc/NF-kappa B pathway [26-37]. Despite that BUF is considered as a promising antitumor agent, its poor water solubility, high cardiac toxicity, short circulation half-life and narrow therapeutic window have limited the clinical application of the drug [26]. 


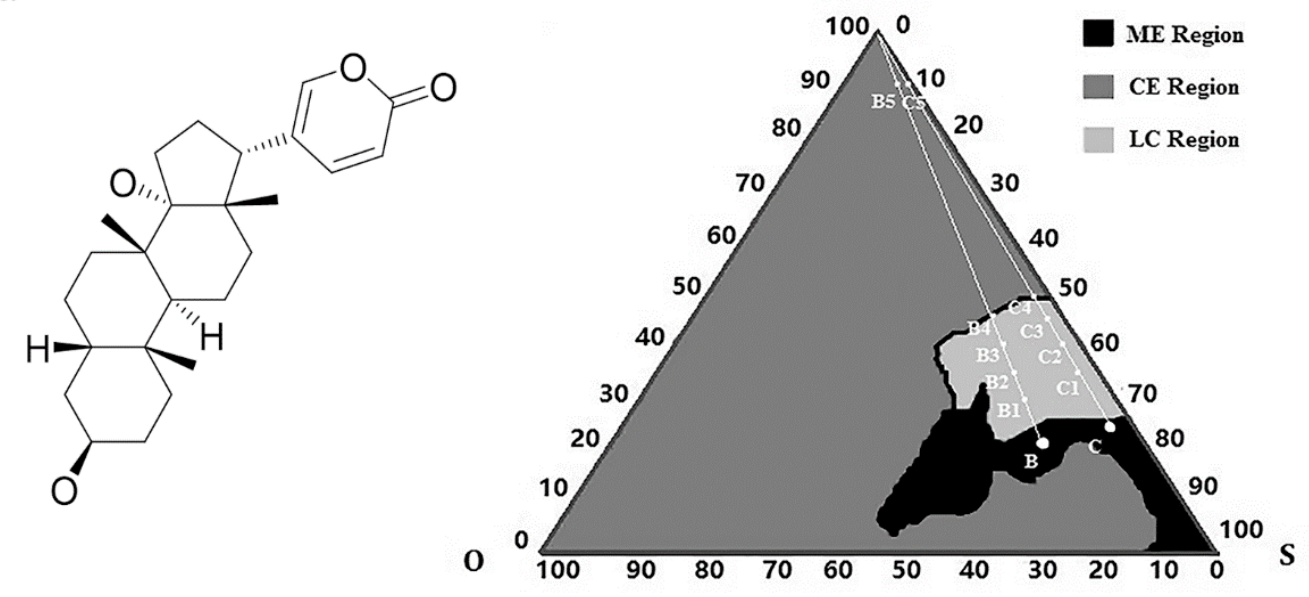

Fig. 1 (a) Chemical structure of the drug bufalin (BUF). (b) A pseudo-ternary phase diagram MCT/HS 15-Span 80/water at $37{ }^{\circ} \mathrm{C}$ [17] with an indication of the investigated oil(O)/surfactant(S)/water(W) compositions along the dilution lines, which are chosen between the regions of microemulsions (ME), liquid crystals (LC), and coarse emulsions (CE). The phase transitions of the $\mathrm{O} / \mathrm{S} / \mathrm{W}$ system from $\mathrm{ME}$ to LC, occurring upon progressive addition of water, are examined by SAXS along the lines B-B5 and C-C5.

Various types of drug delivery systems have been investigated with the purpose to improve the absorption and bioavailability of bufalin in anticancer therapies [33-38]. Liposomes coated with citrus pectin have been employed for encapsulation of BUF [33]. This carrier system has been more stable in comparison to traditional liposomes, but the drug entrapment efficiency was about 49\%. Polymer nanoparticle made of mPEG-PLGA-PLL-cRGD (methoxy polyethylene glycol (mPEG), polylactic-co-glycolic acid (PLGA), poly-L-lysine (PLL), and cyclic arginine-glycine-aspartic acid (cRGD)) have been investigated as a potential delivery system of BUF in antitumor therapy [34]. Bufalin-loaded mPEG-PLGA-PLL-cRGD nanoparticles have been characterized by drug entrapment efficiency (EE) of $(81.7 \pm 0.9) \%$ and drug loading (DL) of $(3.92 \pm 0.2) \%$. The use of biotinylated chitosan has allowed the preparation of BUF-encapsulating nanoparticles with entrapment efficiency of $77.5 \pm 2.5 \%$ [35].Considering the complexity of the reported preparation protocols, their high costs, and the instability of the nanoparticulate formulations, the 
need of a more simple method for BUF vectorization becomes evident together with the requirement for higher stability and drug entrapment efficiency.

Here we exploit the structural features of the in-situ phase transition from a microemulsion to a lyotropic liquid crystal (LC) toward the aim of controlled preparation of bufalin drug-loaded carriers. We use the pseudo-ternary phase diagram of an oil(O)/surfactant(S)/water(W) system containing medium chain capric/caprylic triglycerides (MCT) and a co-surfactant blend of Macrogol (15)-hydroxystearate (Solutol HS 15) and sorbitan monooleate (Span 80) [39] (Fig. 1b). Solutol HS 15 is a safety surfactant, which can be sterilized and used in painless injections at concentrations as high as 50\% (w/w) [40]. All materials employed for the sample preparation are biocompatible, injectable, sterilizable, and available in a nonpyrogenic grade. Moreover, they are non-hemolytic and non-irritating to the nervous system and have been approved by the US Food and Drug Administration (FDA) for intramuscular injections [39]. The mixture of Solutol HS 15 (an O/W emulsifying agent of hydrophilic nature, $\mathrm{HLB}=15)$ and Span 80 (a W/O emulsifying agent of lipophilic nature, $\mathrm{HLB}=4.3$ ) yields an average $\mathrm{HLB}=12.3$ (hydrophilic-lipophilic balance) value, which facilitates the in situ phase transition from ME to LC upon contact with aqueous phase.

The aim of this study is to investigate by SAXS the structural changes occurring along two dilution lines of the MCT/HS 15-Span 80/water pseudo-ternary phase diagram and demonstrate the sustained-release properties of the in situ prepared carriers of the antitumor drug BUF. The rationale of using two surfactant molecules in this work is based on the fact that they can assemble in the mixture as a double chain amphiphile, which spontaneously forms a lamellar phase structures upon hydration. The human lung cancer cell line A549 was employed as a cancer cell model for evaluation of the biological effects of the encapsulated bufalin.

\section{Material and methods}

\subsection{Materials}




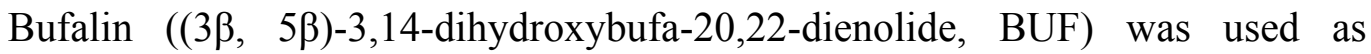
received from Chengdu Herbpurify Co. Ltd (China). The purity was greater than $98 \%$ according to the specification from the supplier. Macrogol (15)-hydroxystearate (Solutol HS 15) was provided by BASF (Germany). Capric/caprylic triglycerides (MCT), sorbitan monooleate (Span 80) and absolute ethyl alcohol were purchased from Aladdin Chemical Reagent Co. Ltd. (China). The water used in the experiment was double distilled.

\subsection{Sample Preparation}

Two compositions of different contents of oil (MCT) were selected from the pseudo-ternary phase diagram reported by Wu et al. [39]. They are shown in Fig.1b as points $\mathbf{B}$ and $\mathbf{C}$ and are referred to as B-LC-ME and C-LC-ME hereafter. Table S1 presents the materials mass ratios in the investigated starting mixtures B-LC-ME and C-LC-ME. The drug BUF was dissolved in absolute ethanol for the preparation of BUF-loaded carriers, which were obtained by the same method. Starting from points $\mathbf{B}$ and $\mathbf{C}$, the water content was increased along the indicated dilution lines (Fig. 1b), which served for the preparation of samples B1, B2, B3, B4, B5, C1, C2, C3, C4, and C5 (Table S1).

\subsection{Structure characterization}

The small-angle X-ray scattering (SAXS) was performed at the beamline BL19U2 of the National Center for Protein Science Shanghai (NCPSS) at Shanghai Synchrotron Radiation Facility (SSRF). Rheological measurements were performed on a AR $2000 \mathrm{EX}$ rheometer (TA Instruments) at $37{ }^{\circ} \mathrm{C}$. The dynamic light scattering (DLS) measurements were performed with a Delsat Nano C Particle Analyzer (Beckman Coulter, USA) to get the mean hydrodynamic diameters and their size distributions. Cryo-transmission electron microscopy studies were done in laboratory for Soft Matter Electron Microscopy, University of Bayreuth. The experimental details were in Electronic Supplementary Information (ESI).

\subsection{Encapsulation efficiency and drug loading}

The encapsulation efficiency (EE) and drug loading (DL) of the BUF drug-loading carriers were determined by the ultrafiltration method [7]. The 
quantitative HPLC analysis method was used to measure the free drug concentration. The wavelength was set at $298 \mathrm{~nm}$ for the HPLC analysis. The EE and DL were estimated using Equations (1) and (2) below:

$$
\begin{aligned}
& \mathrm{EE} \%=\left(1-\frac{C_{F}}{C_{T}}\right) \times 100 \% \\
& \mathrm{DL} \%=\left(\frac{C_{T}-C_{F}}{C_{L}}\right) \times 100 \%
\end{aligned}
$$

where $C_{T}, C_{F}$, and $C_{L}$ are the total amount of BUF present in the dispersion system, the amount of non-entrapped BUF, and the total amount of lipids and encapsulated BUF, respectively.

\subsection{In vitro drug release experiments}

The in vitro release of BUF from LC-ME- BUF was determined by the dialysis method. The freshly prepared LC-ME-BUF samples were placed into dialysis tubes with $14 \mathrm{kDa}$ MW cutoff. The latter were incubated in the release medium (PBS, phosphate buffer saline, $\mathrm{pH} 7.4 ; 150 \mathrm{~mL})$ at $37^{\circ} \mathrm{C}$. Samples $(4 \mathrm{~mL})$ were taken out from the release medium at predetermined time points and the system was refilled with the same volume of fresh medium. Subsequently, the BUF content was measured by the quantitative HPLC analysis method.

\subsection{Cytotoxicity assay}

The viability of A549 cells, treated with free drug BUF, blank (B-LC-ME and C-LC-ME) and BUF-loaded carriers (B-LC-ME-BUF and C-LC-ME-BUF), was measured in vitro using a Cell Counting Kit-8 (CCK-8) assay. For this purpose, the A549 cells were seeded at a density of $5 \times 10^{3}$ cells per well in 96-well plates containing $100 \mu \mathrm{L}$ medium per well. After incubation for $24 \mathrm{~h}$, the culture medium was removed and the cells were exposed for $48 \mathrm{~h}$ to various concentrations of free drug BUF or multicomponent formulations (B-LC-ME-BUF, C-LC-ME-BUF, B-LC-ME and C-LC-ME) dispersed in the medium. Then, $10 \mu \mathrm{L}$ of CCK-8 reagent was added per well. After incubating the cells for another $1 \mathrm{~h}$, the absorbance was recorded at a wavelength of $450 \mathrm{~nm}$ using a Spectra Max M5 instrument. The GraphPad Prism software (Windows version) was used to calculate the IC50 values 
(i.e. the $50 \%$ inhibiting concentration).

\subsection{Cell apoptosis assay}

The effects of free BUF, B-LC-ME-BUF, C-LC-ME-BUF, B-LC-ME and C-LC-ME on the A549 cell apoptosis were assessed using flow cytometry. Briefly, the cells were harvested and suspended at a concentration of $2 \times 10^{5}$ cells/well in six-well plates overnight. Then, they were subjected to different types of treatments (free BUF, B-LC-ME-BUF, C-LC-ME-BUF, B-LC-ME and C-LC-ME) for $48 \mathrm{~h}$. The cells were then collected by centrifugation and washed twice with cold PBS at $1000 \mathrm{rpm}$ for 5 min. Finally, the cells were gently re-suspended in $500 \mu \mathrm{L}$ of binding buffer and incubated with $5 \mu \mathrm{L}$ of Annexin V-FITC and $5 \mu \mathrm{L}$ of PI in dark for $10 \mathrm{~min}$. After incubation, the treated cells were analyzed using a FACScan flow cytometer (Becton Dickinson, USA).

\subsection{Pharmacokinetics}

The bioavailability of the pure BUF was compared to that of BUF incorporated in the C-LC-ME-BUF carriers. Wistar rats were used in these experiments. They were divided into two groups $(\mathrm{n}=6)$. Pure BUF or C-LC-ME-BUF formulations were given to the rats at a dose of $10 \mathrm{mg} / \mathrm{kg}$. After the parenteral administration, approximately $0.5 \mathrm{~mL}$ of blood was collected from the ocular vein into a heparinized tube at times $5,15,30,45,60,90,120,240,360$ and 600 minutes. The collected blood samples were analyzed by HPLC after corresponding treatment. The pharmacokinetic parameters such as clearance (CL) and area under the concentration-time curve (AUC) were calculated using the pharmacokinetic software 3P97 (Mathematics Pharmacological Committee of the Chinese Academy of Pharmacology, Shanghai, People's Republic of China).

\subsection{Statistic analysis}

Data are expressed as mean $\pm \mathrm{SD}$ and were analyzed with the student's t-test. The difference between two means was considered statistically significant when $P<0.05$.

\section{Result and discussion}




\section{Structural characterization of the LC-ME and LC-ME-BUF systems}

The multicomponent B-LC-ME and C-LC-ME mixtures were homogeneously formed upon physical agitation. The principal difference between the B-LC-ME and C-LC-ME compositions was in the incorporated oil amount. The water content was systematically varied along the lines $\mathbf{B}-\mathbf{B} 4$ and $\mathbf{C}-\mathbf{C} 4$, which are shown in the pseudo-ternary phase diagram in Fig. 1b. Structural characterizations were performed by SAXS for the oil/surfactant/water compositions depicted in Table S1.

The SAXS results for the samples of varying water contents are presented in Fig. 2. They indicate that the B-LC-ME and C-LC-ME systems were both of microemulsion organization (points $\mathbf{B}$ and $\mathbf{C}$ in Fig. 1b) before the onset of their dilution by water along the lines $\mathbf{B}-\mathbf{B} 4$ and $\mathbf{C}-\mathbf{C} 4$.
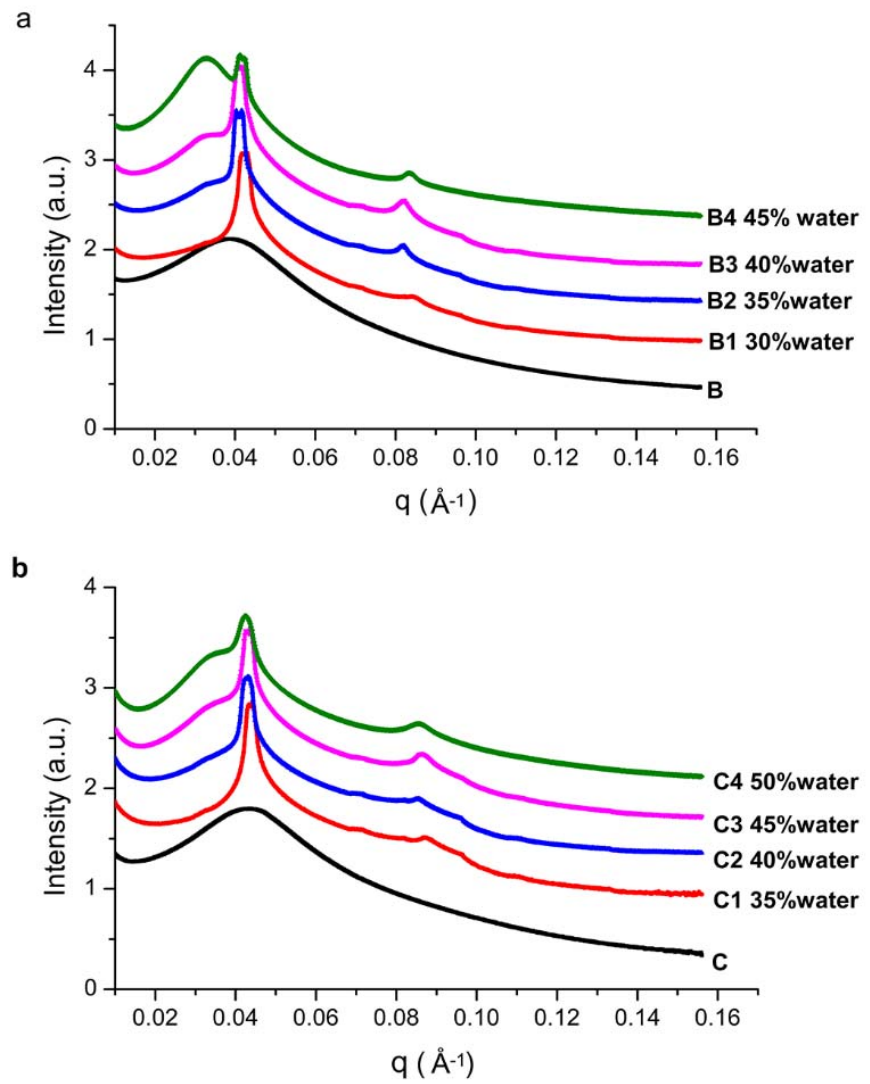

Fig. 2 SAXS patterns of B-LC-ME (panel (a)) and C-LC-ME systems (panel (b)) recorded at increasing water content along the corresponding lines of the pseudo-ternary phase diagram (Fig. 1b, Table S1). The water contents of the samples 
are indicated for every plot in the figure.

The smooth black curves in Fig. 2a (sample B) and Fig. 2b (sample C) display correlation peak maxima in the SAXS patterns. The latter are typical for microemulsion-type oil/surfactant/water systems as well as for sponge-type lipid/water systems $[42,43]$. The more hydrated samples B1 to B4 and C1 to C4 display first and second orders of Bragg diffraction peaks and therefore characterize a lamellar structure or a phase of multilamellar onions (spacing ratio of 1:2:3) $[23,42,43]$. The $q$-vector values for the Bragg diffraction maxima in sample B1 are equal to $q_{1}=0.042 \AA^{-1}$ and $q_{2}=0.084 \AA^{-1}$. The Bragg peak maxima of the lamellar liquid crystalline structure determine the interlayer repeat distance, $d$, through the relationship $d=2 \pi . n / q$, where $n$ is the order of the Bragg peak. This equation yielded a repeat spacing value $d=14.9 \mathrm{~nm}$ for the multilamellar onions generated in sample B1. Therefore, the SAXS results evidence a phase transition from a microemulsion phase to a lamellar liquid crystalline structure in the investigated multicomponent amphiphilic mixtures. Considering that the recorded Bragg peaks are less intense as compared to those for a well-ordered lamellar phase at this hydration level, it may be suggested that multilamellar onion vesicles are formed under such conditions. In addition, one observes a tendency of emergence of an additional peak at lower $q$-vectors with the increase of the water content. This implies a coexistence of the multilamellar structures with some microemulsion content (samples B3, B4, C3 and C4) upon approaching the phase boundary (Fig. 1b) [44].

The comparison of the SAXS patterns for samples $\mathbf{B} \mathbf{1}$ and $\mathbf{C 1}$ is done in Fig. 3a. It demonstrates that both samples are of lamellar structures. The repeat distance, $d$, was found to be larger for sample B1, for which the Bragg peaks maxima were positioned at lower $q$-vector values. With the increase of the oil content in the lamellar structures (from $\mathbf{C 1}$ to B1), the interaction between the aqueous layers is reduced because the augmented oil content increases the distance between the aqueous 
channels. This will decrease the electrostatic or dipole-dipole interactions among the aqueous channels, which is inversely proportional to the distance. This effect can be due to the thickening of the oil reservoirs created by the hydrophobic moieties of the surfactant molecules. The arising structural difference in the packing of the two multilamellar structures may provoke differences in their controlled release properties. These effects will be investigated below.
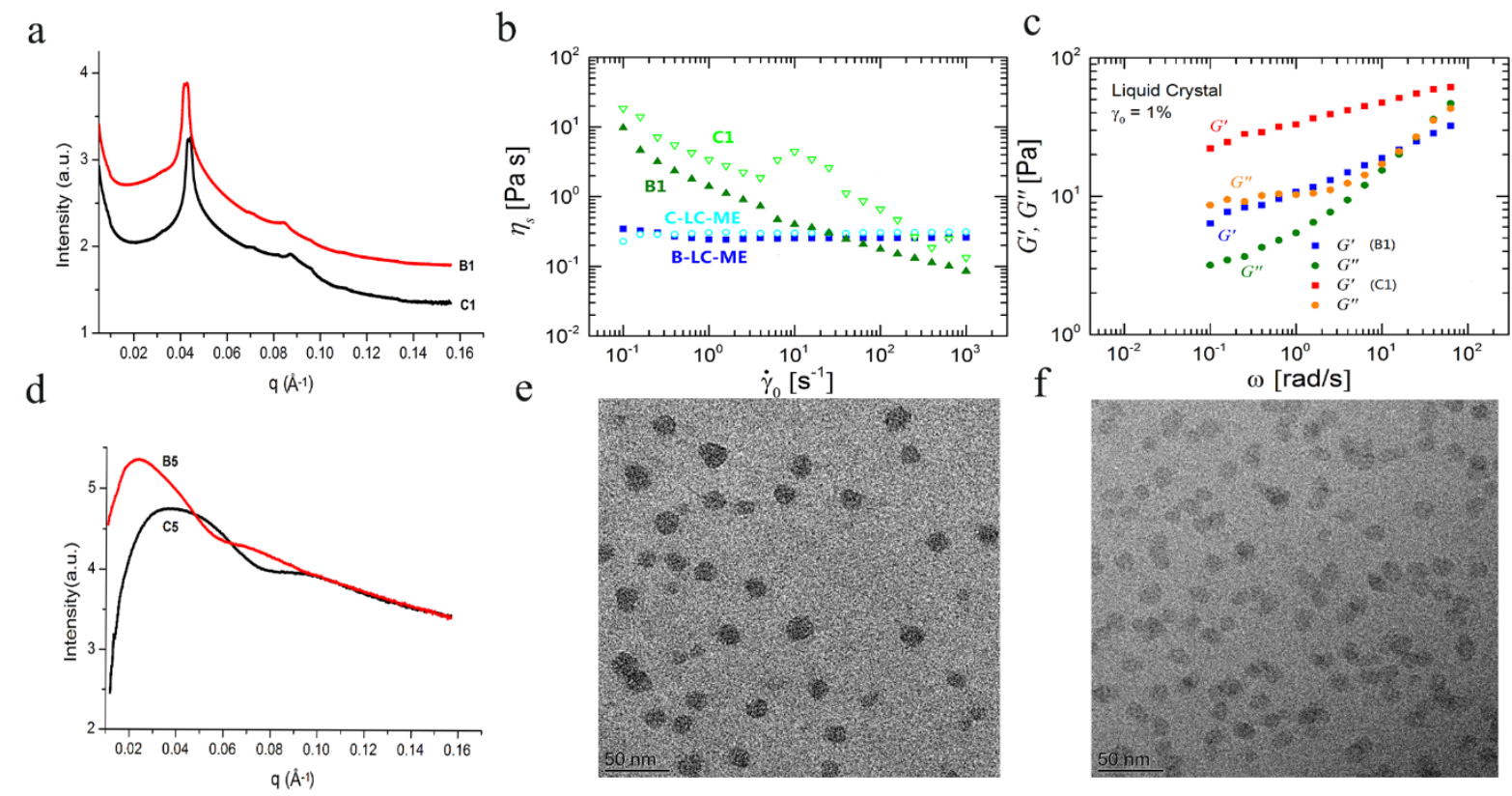

Fig. 3. Comparison of the SAXS patterns of samples B1 and C1 (a) and of B5 and C5 (d). Cryo-TEM images of sample B5 (e) and C5 (f). Dynamic viscosity (b) and energy storage modulus (c) of samples B1 and $\mathbf{C 1}$.

The structural features of samples containing high water content $\sim 90 \% \mathrm{w} / \mathrm{W}$ (points B5 and $\mathbf{C 5}$ belonging to the $\mathrm{CE}$ region of the diagram in Fig. 1b) are also compared. The scattering curves in Fig. 3d revealed that the correlation peak maximum of sample $\mathbf{B 5}$ is shifted to a lower $q$-vector value with regard to that of 
sample C5. This implies that the distances between the surfactant aggregates in the aqueous dispersion should be larger. Additional repulsion is required to keep the droplets apart at larger distance. This output was verified by DLS experiments as well as by Cryo-TEM imaging of the studied dispersions. The particle size measurements by DLS established that sample B5 exhibits a mean particle diameter $d_{H}=24.3 \pm 0.8 \mathrm{~nm}$. Indeed, the diameter was slightly bigger than that determined for sample C5, $d_{H}=18.6 \pm 0.5 \mathrm{~nm}$.

The performed cryo-TEM analysis confirmed the SAXS and DLS results. Fig. 3 (e and f) clearly shows that samples B5 and C5 are constituted by microemulsions of spherical droplets. The latter are homogeneously dispersed and display small particle sizes (below $25 \mathrm{~nm}$ ). The comparison performed at a same magnification scale revealed that the mean droplet sizes in samples B5 and C5 slightly differ as determined above.

The structural characterization of the drug-loaded carriers by SAXS established that the BUF encapsulation has a pronounced influence on the scattering curves of the B-LC-ME-BUF and C-LC-ME-BUF systems. Representative SAXS patterns for the different structural organizations, corresponding to the $\mathrm{ME}, \mathrm{LC}$ and $\mathrm{CE}$ regions of the diagram, are shown in Fig. 4. The obtained SAXS plots demonstrate that the internal structures of the drug-loaded carriers are preserved after BUF encapsulation. However, the positions of the Bragg peaks (or the correlation peaks bumps) are displaced in the direction of smaller $q$-vector values. This indicates that the interlayer spacings of the samples increase to different degrees depending on the structural type of the blank 
carriers (ME, LE or CE). At ME region, maximum peak of B-LC-ME becomes sharper after loading BUF, it points that the interaction between ME increases, which may due to excluded volume effect. For C-LC-ME there is an opposite phenomenon after loading BUF, maximum peak becomes blunt and the peak value moved to smaller $q$, which means a larger distance and a lower concentration of droplets. At LC region, the repeat distance of B-LC-ME and C-LC-ME are all increase and peaks become smaller, less ordering after loading BUF. These data evidenced that the BUF molecules are successfully encapsulated, and thus the structural parameters of the host carrier systems are modified. Taking into account the considerable modification of the SAXS patterns in Fig. 4, a high degree of BUF encapsulation in these samples can be suggested. This conclusion is supported also by the direct determination of the entrapment efficiency of BUF in these carriers (see the section below). 

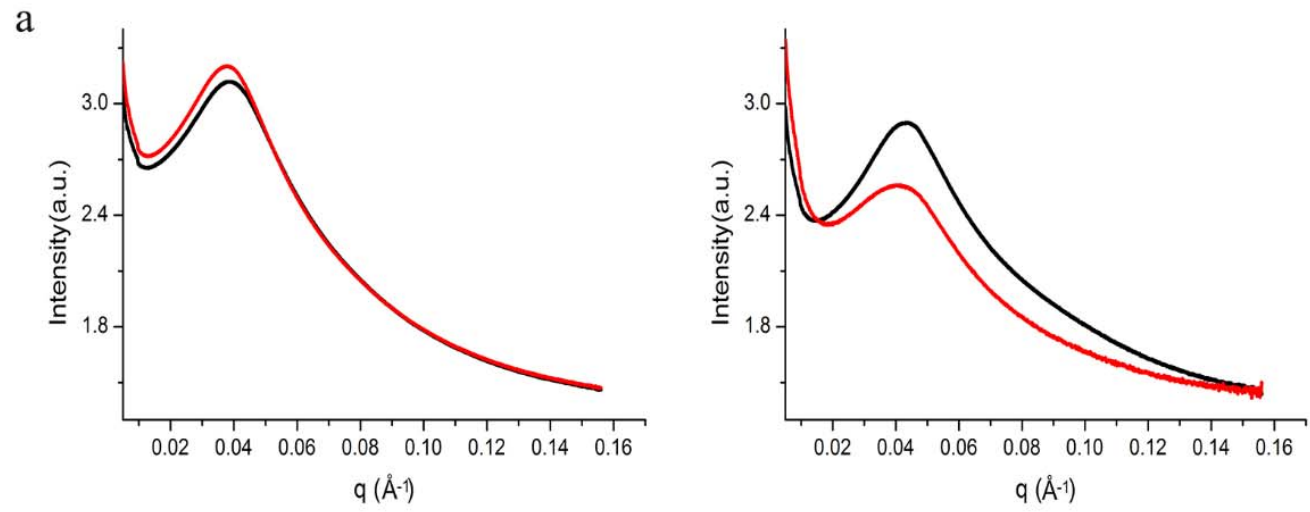

b
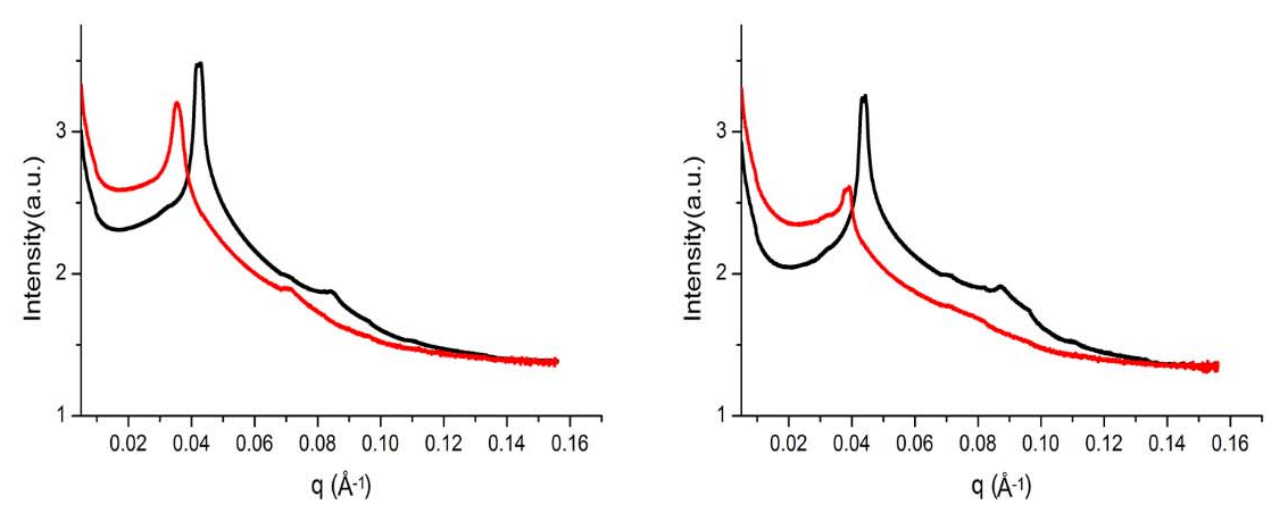

$\mathrm{c}$
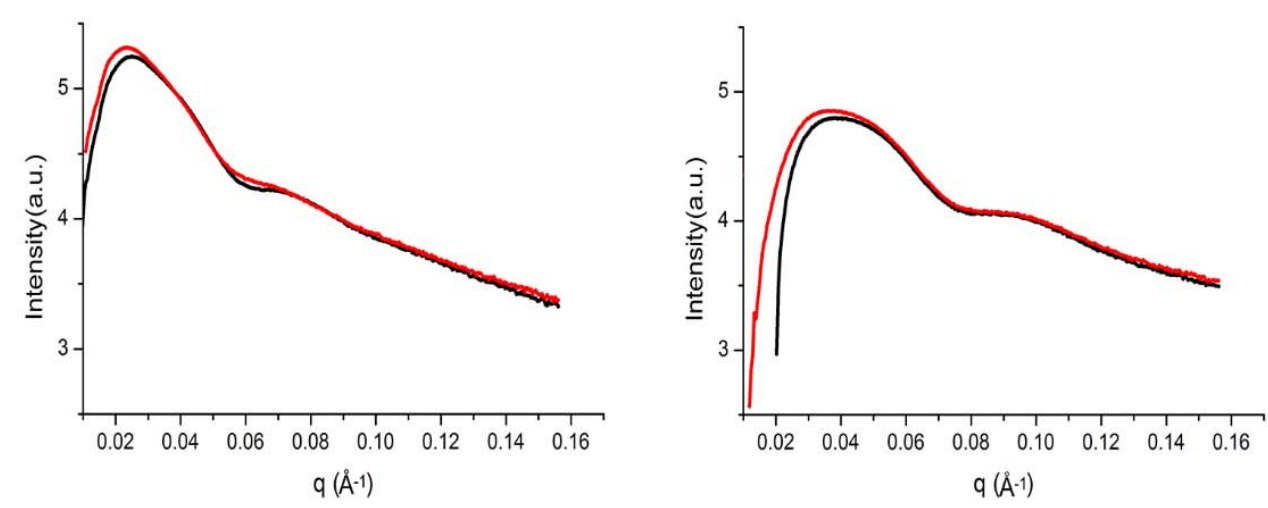

Fig. 4. SAXS patterns of BUF drug-loaded carriers (red plots): B-LC-ME-BUF (left column) and C-LC-ME-BUF (right column) versus blank carriers (black plots) characteristic of the examined three regions of the pseudo-ternary phase diagram: microemulsion region ME (a), liquid crystal LC region (b), and coarse emulsion region $\mathrm{CE}(\mathrm{c})$.

It should be noted that bufalin was dissolved in ethanol for the preparation of drug-loaded carriers. The recommended dosage of ethanol injection is 5-10\% [45]. The maximum content in these samples is $7.8 \%$, which is within the safe range. Ethanol, included in the formulations, may not only improve the solubility of the drug 
BUF, but also contribute to the formation and stabilization of lyotropic liquid crystalline phases. Ethanol may penetrate into the surfactant membrane, modify its organization, increase its fluidity and reduce the system viscosity [41]. The above data show that the structural parameters of the B-LC-ME-BUF and C-LC-ME-BUF liquid crystalline structures are more sensitive to the BUF loading as compared to the water-diluted CE dispersions.

\subsection{Encapsulation efficiency, drug loading, and rheological characterization of}

\section{the carriers in relation to bufalin release}

Table S2 shows the encapsulation efficiency (EE) and drug loading (DL) values determined for freshly prepared B-LC-ME-BUF and C-LC-ME-BUF carriers. We found that the EE values for BUF entrapment are superior to $90 \%$. No significant changes in the drug loading and drug entrapment efficiencies were observed for the investigated compositions during 90 days of carrier storage. The high EE and DL of BUF in the LC-ME carriers should be related to its lipophilic nature and its affinity for the membrane compartment reservoirs of the lyotropic lamellar phase.

The release profiles of BUF from the B-LC-ME-BUF and C-LC-ME-BUF carrier systems are shown in Fig. 5. The release of BUF from the free-drug suspension was investigated as a control. Fig. 5 demonstrates that more than $90 \%$ of BUF was rapidly released from the pure drug suspension within $12 \mathrm{~h}$. The B-LC-ME-BUF and C-LC-ME-BUF formulations showed smooth release curves of a similar trend. In this case, no burst release was observed from the B-LC-ME-BUF and C-LC-ME-BUF carriers. The obtained result is consistent with their high encapsulation efficiency. We established that the release rate of BUF from the C-LC-ME-BUF formulation was 
slower than that from B-LC-ME-BUF. The cumulative release of BUF in the C-LC-ME-BUF system was only $48.3 \pm 2.8 \%$ after $96 \mathrm{~h}$. The corresponding value for the B-LC-ME-BUF sample was $67.9 \pm 3.1 \%$. The release curve of bufalin follow the square root of time kinetics (Fig. S2) showed a linear relationship. Generally it suggested the mesophases have a diffusion controlled release rate [48]. The established sustained release behavior is in agreement with the results obtained in the structural SAXS (Fig. 4) and the rheological (Fig. 3) experiments.

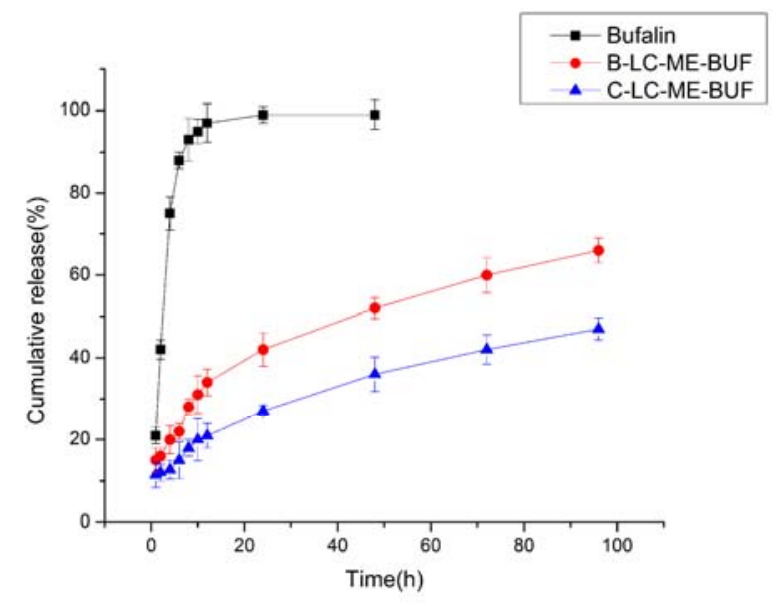

Fig. 5. Cumulative in vitro release of bufalin (BUF) from aqueous suspension (squares) and from B-LC-ME-BUF (circles) and C-LC-ME-BUF carriers (triangles) at $37^{\circ} \mathrm{C}$.

For better understanding of the different controlled release profile of the investigated carriers, we considered the arguments of Wu et al. [17] who has pointed out the role of the different viscosities of the multicomponent systems. The authors have emphasized that the amphiphilic compositions may enable the formation of different LC structures such as lamellar $(\mathrm{L} \alpha)$, cubic and hexagonal $\left(\mathrm{H}_{\mathrm{II}}\right)$ mesophases, which are characterized by different rheological properties influencing the drug release profiles [17]. 
Taking into account that the structures of the B-LC-ME series, shown in Fig. 2, are similar to those of the C-LC-ME samples, we proceeded to additional experiments focusing on the rheology of the two groups of formulations. Samples B1 and C1 were selected for rheological experiments because they involved the same hydration levels, in same aqueous medium, and were both prepared from the ME region of the diagram. The SAXS results revealed that these two samples have liquid crystalline structures (Fig. 3a).

Fig. 3(b and c) presents the rheological data obtained for samples B1 and C1 of the B-LC-ME and C-LC-ME series, respectively. They indicate that the B-LC-ME and C-LC-ME carriers, prepared from the microemulsion region, are Newtonian fluids. The determined dynamic viscosities are in the range of few mPa-s (Fig. 3b), which evidences that these formulations are appropriate for injection use [17].

At variance, samples B1 and $\mathbf{C 1}$, prepared in the lyotropic liquid crystal (LC) region, showed non-Newtonian fluid properties. They were characterized by significantly higher viscosities as compared to the MEs formulations. Despite the similarity of the condensed phase multilamellar structures, the viscosity of sample $\mathbf{C 1}$ was higher than that of sample B1. Both carriers exhibited shear-thinning properties (Fig. 3b and 3c). The viscosities decreased exponentially upon increasing the shear rate. It is worth noting that sample $\mathbf{C 1}$ displayed shear-thinning behavior at low shear rates. However, shear thickening phenomenon was observed to a certain extent when the shear rate increased. This may be due to changes in the internal structure of the liquid crystalline phase upon application of the shear stress, which may result in 
multilayer vesicle formation [23]. Fig. S1 present the curve of shear stress curve as a function of the shear rate B-LC-ME, C-LC-ME C1and B1.

The established dynamic rheological properties of samples B1 and C1 demonstrated typical characteristics for a lamellar LC phase [23]. The energy storage (elastic) modulus $\left(G^{\prime}\right)$ was greater than the loss (viscous) modulus $\left(G^{\prime \prime}\right)$ at low shear stresses. At higher ones, the energy storage modulus of sample $\mathbf{C 1}$ was similar to that of B1 (Fig. 3c). However, the strain of sample $\mathbf{C 1}$ was obviously higher than that of sample B1. It may be suggested that this effect may be relevant to the differences in the drug release profiles of the carriers[47].

3.3 Biological evaluation of the carriers in cell experiments and in vivo pharmacokinetics

Fig. 6a presents the cytotoxicity of the investigated formulations (B-LC-ME-BUF, C-LC-ME-BUF, blank B-LC-ME and C-LC-ME carriers, and free BUF drug), which was evaluated with the A549 cell. The obtained data showed that the blank B-LC-ME and C-LC-ME carriers have no obvious toxicity. This implies that the cell death, resulting from the B-LC-ME-BUF and C-LC-ME-BUF treatments, should be primarily due to the BUF release. Besides, the cytotoxic effect of BUF on the A549 cells was enhanced after its incorporation in the LC-ME carriers. The improved penetration of BUF into the cells and the enhanced intracellular drug accumulation may be explained by the fact that LC-ME may carry the drug into the cancer cells through endocytosis. Therefore, the B-LC-ME-BUF and C-LC-ME-BUF 
formulations should have high potential for use in cancer therapy. The $\mathrm{IC}_{50}$ values of every experimental group are summarized in Table S3. BUF encapsulated in C-LC-ME displayed the lowest $\mathrm{IC}_{50}$ value. This result suggests that the C-LC-ME-BUF formulation may significantly improve the bioavailability of BUF.

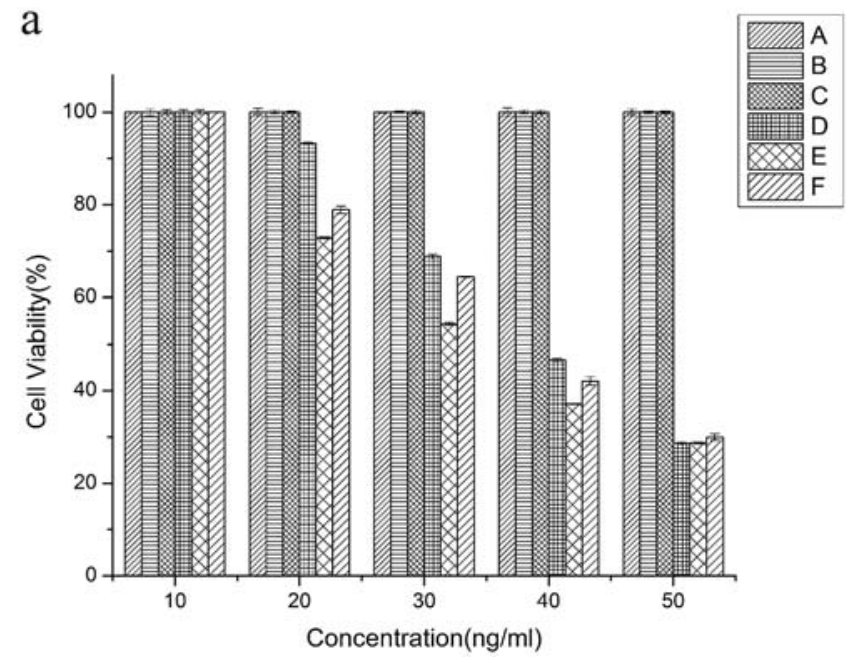

b
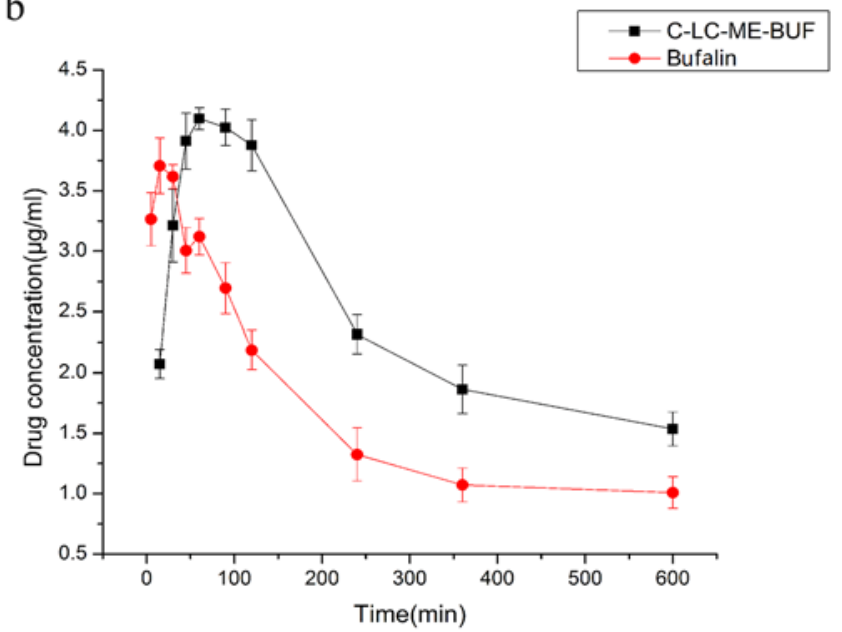

Fig. 6. (a) In vitro cytotoxicity of BUF, B-LC-ME-BUF and C-LC-ME-BUF determined with human lung carcinoma A549 cells (A: control group, B: B-LC-ME, C: C-LC-ME, D: BUF, E: C-LC-ME-BUF, F: B-LC-ME-BUF); (b) Plasma concentration versus time curves for C-LC-ME-BUF and free BUF; $P<0.05$.

Apoptosis represents an important mechanism for the inhibition of cancer cell proliferation by anticancer agents. The apoptotic profile of free BUF, B-LC-ME-BUF and C-LC-ME-BUF in A549 cells was evaluated by flow cytometry. Table 1 
demonstrates that the C-LC-ME-BUF induced enhanced apoptosis of the A549 cells (53.97\%) as compared to the B-LC-ME-BUF formulation $(39.44 \%)$ and the free drug (BUF) suspension (37.15\%). The data show that the free BUF induces apoptosis of the A549 cells at the same extent as the B-LC-ME carriers. However, the apoptosis rate was markedly increased for BUF encapsulated in the C-LC-ME carriers. This might be due to the better uptake and the greater accumulation of BUF into A549 cells in a C-LC-ME encapsulated state. The performed biological tests revealed that the C-LC-ME-BUF carriers exerted a greater effect on the A549 cells as compared to free BUF and the B-LC-ME-BUF formulation. This finding gives a priority to C-LC-ME as a suitable drug delivery system of BUF.

Table 1. Apoptosis rate of A549 cells determined at drug concentration of $7 \mathrm{ng} / \mathrm{ml}$.

\begin{tabular}{lcc}
\hline & Apoptosis rate $(\%)$ & Survival rate $(\%)$ \\
\hline Free BUF drug & 37.15 & 60.53 \\
B-LC-ME-BUF & 39.44 & 58.46 \\
C-LC-ME-BUF & 53.97 & 45.08 \\
\hline
\end{tabular}

Based on the obtained results from the in vitro cell culture experiments, the C-LC-ME-BUF carriers were selected for in vivo pharmacokinetic experiments with rats through intragastric administration. We examined the sustained release capacity of the C-LC-ME-BUF. The plasma concentration versus time curves for C-LC-ME-BUF and free BUF are presented in Fig. 6b, which was in the safe range of bufalin [46]. 
The pharmacokinetic parameters examined in this work are summarized in Table 2. The sustained release system C-LC-ME-BUF was compared with the free drug. The values of the parameters $\mathrm{AUC}_{0-\tau}, \mathrm{MRT}$ and $\mathrm{t}_{1 / 2}$ were $1.39,1.83$ and 2.15 times higher for C-LC-ME-BUF than those for the free BUF. The fact that $\mathrm{C}_{\max }$ and $\mathrm{T}_{\max }$ of C-LC-ME-BUF were higher than those of the control group (BUF) indicated that the initial burst release can be significantly reduced by the formulation. The C-LC-ME-BUF carrier produced highest blood concentrations evidently due to the fact that C-LC-ME-BUF is able to slowly release the drug.

The high $\mathrm{AUC}_{0-\tau}, \mathrm{AUC}_{0-\infty}$ and $\mathrm{t}_{1 / 2}$ values indicated that C-LC-ME-BUF has a higher consumption rate than the free BUF. Therefore, loading of BUF in C-LC-ME appears to be efficient in prolonging the retention time of BUF in the blood. Despite that the C-LC-ME showed good sustained release in the in vitro release experiment, it was not consistent with the in vivo pharmacokinetic experiments. The possible reason might be the formation of a liquid crystalline structure, which plays a key role in the sustained release and shear-thinning properties. The shear rate from the animal movement may lower the viscosity and increase the biodistribution of the bufalin drug.

Table 2. Pharmacokinetic parameters determined for C-LC-ME-BUF carriers with regard to the free drug BUF.

\begin{tabular}{ccccccc}
\hline & $\begin{array}{c}\text { Cmax } \\
(\mu \mathrm{g} / \mathrm{mL})\end{array}$ & $\begin{array}{c}\text { Tmax } \\
(\mathrm{min})\end{array}$ & $\begin{array}{c}\mathrm{t}_{1 / 2} \\
(\mathrm{~min})\end{array}$ & $\begin{array}{c}\text { MRT } \\
(\mathrm{h})\end{array}$ & $\begin{array}{c}\text { AUC 0- } \tau \\
(\mu \mathrm{g} / \mathrm{mL} \cdot \mathrm{h})\end{array}$ & $\begin{array}{c}\text { AUC 0- } \\
(\mu \mathrm{g} / \mathrm{mL} \cdot \mathrm{h})\end{array}$ \\
\hline C-LC-ME-BUF & 4.10 & 1 & 7.87 & 11.11 & 22.28 & 27.60 \\
BUF & 3.71 & 0.25 & 3.66 & 6.05 & 15.97 & 27.44 \\
\hline
\end{tabular}

Notes: $\tau$, the end time of the dosage interval.

\section{Conclusion}

In this work, we identified conditions for fabrication of sustained-release formulations of the poorly soluble anticancer drug bufalin. The in situ occurring phase 
transition from microemulsions to lamellar liquid crystalline structures, underlying the preparation of the BUF drug-loaded carriers, was characterized by SAXS. Optimal drug loading was achieved for the C-LC-ME-BUF system. The latter provided high encapsulation efficiency and drug loading, long-term stability, and sustained-release properties. The $\mathrm{IC}_{50}$ values, determined in cytotoxicity experiments with human lung carcinoma A549 cells, proved 1.4 times enhancement of the cytotoxicity exerted by the C-LC-ME-BUF carriers in comparison to that of the pure BUF suspension. In addition, the C-LC-ME-BUF carriers considerably increased the number of apoptotic cancer cells. This might result from the improved bioavailability of BUF encapsulated in C-LC-ME as compared to the BUF drug alone. Pharmacokinetic experiments with rats showed that bufalin loaded in C-LC-ME carriers can prolong the retention time of the drug in the blood. In perspective, the established sustained-release drug profiles present interest for in vivo evaluation of the anticancer effects of the C-LC-ME-BUF systems.

\section{Acknowledgement:}

We gratefully acknowledge the support of this work by the National Natural Science Foundation of China (Grant No. 21573070). Also thanks a lot for the support and help of animal experiment from Jianwen Liu. 


\section{References}

[1] Z. Wu, D. Hassan, J.P. Shaw, In-vitro prediction of bioavailability following extravascular injection of poorly soluble drugs: an insight into clinical failure and the role of delivery systems, J. Pharm. Pharmacol 65 (2013) 1429-1439.

[2] Y. Shi, L. Li, Current advances in sustained-release systems for parenteral drug delivery, Expert Opin. Drug Deliv. 2 (2005) 1039-1058.

[3] A.H. Zou, Y.W. Li, Y.Y. Chen, A. Angelova, V.M. Garamus, N. Li, Self-assembled stable sponge-type nanocarries for Brucea javanica oil delivery, Colloids and Surfaces B: Biointerfaces 153 (2017) 310-319.

[4] A. Yaghmur, O. Glatter, Characterization and potential applications of nanostructured aqueous dispersions, Adv. Colloid Interface Sci. 147-148 (2009) 333-342.

[5] X. Mulet, B.J. Boyd, C.J. Drummond, Advances in Drug Delivery and Medical Imaging Using Colloidal Lyotropic Liquid Crystalline Dispersions, J. Colloid Interface Sci. 393 (2013) 1-20.

[6] B. Angelov, V.M. Garamus, M. Drechsler, A. Angelova, Structural analysis of nanoparticulate carriers for encapsulation of macromolecular drugs, J. Mol. Liq. 235 (2017) 83-89.

[7] A. Angelova, V.M. Garamus, B. Angelov, Y.W. Li, A.H. Zou, Advances in structural design of lipid-based nanoparticle carriers for delivery of macromolecular drugs, Adv. Colloid Interface. DOI: 10.1016/j.cis.2017.04.006.

[8] K. Cerpnjak, A. Zvonar, M. Gasperlin, F. Vrecer, Lipid-based systems as a promising approach for enhancing the bioavailability of poorly water-soluble drugs, Acta Pharmaceutica 63 (2013) 427-445.

[9] C.X. He, Z.G. He, J.Q. Gao, Microemulsions as drug delivery systems to improve the solubility and the bioavailability of poorly water-soluble drugs, Expert Opin. Drug Deliv. 7 (2010) 445-460.

[10]A.A. Date, M.S. Nagarsenker, Parenteral microemulsions: an overview, Int. J. Pharm. 355 (2008) 19-30.

[11]C.V. Kulkarni, R. Mezzenga, O. Glatter, Water-in-oil nanostructured emulsions: towards the structural hierarchy of liquid crystalline materials, Soft Matter 6 (2010) 5615-5624.

[12] S.P. Callender, J.A. Mathews, K. Kobernyk, S.D. Wettig, Microemulsion utility in pharmaceuticals: Implications for multi-drug delivery, Int. J. Pharm. 526 (2017) 425-442.

[13] S. Gupta, S.P. Moulik, Biocompatible microemulsions and their prospective uses in drug delivery, Journal of Pharmaceutical Sciences 97 (2008) 22-45.

[14]R.P. Bagwe, J.R. Kanicky, B.J. Palla, D.O. Shah, Improved drug delivery using microemulsions: Rationale, recent progress, and new horizons, Critical Reviews in Therapeutic Drug Carrier Systems, 18 (2001) 77-140.

[15]F.H. Xavier-junior, C. Vauthier, A.R.V. Morais, E.S.T. Egito, Microemulsion systems containing bioactive natural oils: an overview on the state of the art, 
Drug Dev. Ind. Pharm. 43 (2017) 700-714.

[16]J. Chan, G.M.M.E Maghraby, J.P. Craig, R.G. Alany, Phase transition water-in-oil microemulsions as ocular drug delivery systems: in vitro and in vivo evaluation, Int J Pharm. 328(1) (2007) 65-71.

[17]Z. Wu, R.G. Alany, N. Tawfeek, D. Svirskis, A study of microemulsions as prolonged-release injectables through in-situ phase transition, J. Control. Release 174 (2014) 188-194.

[18]X. Ren, D. Svirskis, R.G. Alany, Z. Wu, In-situ phase transition from microemulsion to liquid crystal with the potential of prolonged parenteral drug delivery, Int. J. Pharm. 431 (2012) 130-137.

[19]J. Phelps, M.V. Bentley, L.B. Lopes, In situ gelling hexagonal phases for sustained release of an anti-addiction drug, Colloids and Surfaces B: Biointerfaces 87 (2011) 391-398.

[20]Y. Chen, X. Liang, P. Ma, S. Gui, Phytantriol-Based In Situ Liquid Crystals with Long-Term Release for Intra-articular Administration, AAPS PharmSciTech. 16 (2015) 846-854.

[21]C.C. Mueller-Goymann, S.G. Frank, Interaction of lidocaine and lidocaine- $\mathrm{HCl}$ with the liquid crystal structure of topical preparations, Int. J. Pharm. 29 (1986) 147-159.

[22] C.M. Chang, R. Bodmeier, Low viscosity monoglyceride-based drug delivery systems transforming into a highly viscous cubic phase, Int. J. Pharm. 173 (1998) 51-60.

[23] J. Zipfel, P. Lindner, M. Tsianou, P. Alexandridis, W. Richtering, Shear-induced formation of multilamellar vesicles (Onion) in block copolymers, Langmuir 15 (1999) 2599-2602.

[24]F.A. Plamper, W. Richtering. Functional Microgels and Microgel Systems, Acc. Chem. Res. 50 (2017) 131-140.

[25]H.B. Nirmal, S.R. Bakliwal, S.P. Pawar, In-Situ gel: New trends in controlled and sustained drug delivery system, Int.J. PharmTech Res. 2 (2010) 1398-1408.

[26]T. Song, X. Chu, X. Zhang, L. Chu, Bufalin, a bufanolide steroid from the parotoid glands of the Chinese toad, inhibits L-type $\mathrm{Ca} 2+$ channels and contractility in rat ventricular myocytes, Fundamental and Clinical Pharmacology 31 (2017) 340-346.

[27]C.H. Lee, Y.L. Shih, M.H. Lee, J.G. Chung, Bufalin Induces Apoptosis of Human Osteosarcoma U-2 OS Cells through Endoplasmic Reticulum Stress, Caspase and Mitochondria-Dependent Signaling Pathways, Molecules 22 (2017) 437.

[28]X. Liu, X.Y. Xiao, Q.Y. Shou, J.C. Wang, Bufalin inhibits pancreatic cancer by inducing cell cycle arrest via the c-Myc/NF-kappa B pathway, Journal of Ethnopharmacology 193 (2016) 538-545.

[29] S.H. Wu, D.T. Bau, Y.T. Hsiao, J.G. Chung, Bufalin induces apoptosis in vitro and has antitumor activity against human lung cancer xenografts in vivo, Environ Toxicol. 32 (2017) 1305-1317.

[30] J.Y. Yeh, W.J. Huang, S.F. Kan, P.S.Wang, Effects of bufalin and cinobufagin on 
the proliferation of androgen dependent and independent prostate cancer cells Prostate 54 (2003) 112-124.

[31]D. Li, X. Qu, K. Hou, Y. Liu, PI3 K/Akt is involved in bufalin-induced apoptosis in gastric cancer cells, Anticancer Drugs 20 (2009) 59-64.

[32]Z.T. Yuan, X.J. Shi, Y.X. Yuan, Y.Y. Qiu, P.H. Yin, Bufalin reverses ABCB1-mediated drug resistance in colorectal cancer, Oncotarget 8 (2017) $48012-48026$.

[33]Y. Li, H. Zhao, L.R. Duan, S.W. Wang, Preparation, characterization and evaluation of bufalin liposomes coated with citrus pectin, Colloids Surfaces A 444 (2014) 54-62.

[34]P. Yin, Y. Wang, Y. Qiu, Q. Li, Bufalin-loaded mPEG-PLGA-PLL-cRGD nanoparticles: preparation, cellular uptake, tissue distribution, and anticancer activity, International Journal of Nanomedicine 7 (2011) 3961-3969.

[35]X. Tian, H. Yin, S. Zhang, J. Fang, Bufalin loaded biotinylated chitosan nanoparticles: An efficient drug delivery system for targeted chemotherapy against breast carcinoma, Eur. J. Pharm. Biopharma. 87 (2014) 445-453.

[36]H. Zhang, G. Yang, L. Qing, Y. Su, C. Ling, Pharmacokinetic, biodistribution, and antihepatocarcinoma effect studies of bufalin-loaded bovine serum albumin nanoparticles, Nanomedicine Nanotechnology Biology and Medicine 12 (2016) 536-536.

[37] S.J. Zhang, Y.T. Zhang, J.H. Zhao, V.P. Feng, Preparation and in vitro anti-tumor properties of toad venom extract-loaded solid lipid nanoparticles, Die Pharmazie 68 (2013) 653-660.

[38]T. Liu, X. Yuan, T. Jia, C. Liu, Y. Yuan, Polymeric prodrug of bufalin for increasing solubility and stability: synthesis and anticancer study in vitro and in vivo, Int. J. Pharm. 506 (2016) 382-393.

[39] K. Hans, Handbook of Liquid Crystals, Verlag Chemie, Weinheim, USA 1980.

[40]R.G. Strickley, Solubilizing excipients in oral and injectable formulations, Pharmaceutical Research 21 (2004) 201-230.

[41]A.C. Williams, B.W. Barry, Penetration enhancers Adv. Drug Deliv. Rev. 56 (2004) 603.

[42]B. Angelov, A. Angelova, U. Vainio, V.M. Garamus, P. Couvreur, Long living intermediates during a lamellar to a diamond-cubic lipid phase transition: A small-angle X-ray scattering investigation, Langmuir 25 (2009) 3734-3742.

[43]K. Brandenburg, W. Richter, M.H. Koch, U. Seydel, Characterization of the nonlamellar cubic and HII structures of lipid A from Salmonella enterica serovar Minnesota by X-ray diffraction and freeze-fracture electron microscopy, Chemistry \& Physics of Lipids 91 (1998) 53-69.

[44]Kegel W K, Lekkerkerker H N W. Competition between a lamellar and a microemulsion phase in an ionic surfactant system[J]. Journal of Physical Chemistry 97 (1993) 11124-11133.

[45] Raymond C Rowe, Paul J Sheskey and Sian C Owen. Handbook of Pharmaceutical Excipients. Fourth Edition. London, Chicago: Pharmaceutical Press, 2003. 
[46] Study on in situ intestinal absorption of bufalin in rats. Y. Liu, N. Feng, J. Xu, S. Wu, X, Zhang, Lishizheng Medicine and Materia Research, 20 (2009) 428-430.

[47]X. Liu, Z. Wang, L. Wei, Curcumin-Encapsulated Hexagonal Liquid Crystalline Formed by Brij 97/NaDC Mixtures, Journal of Dispersion Science \& Technology 35 (2014) 1699-1708.

[48]Clogston J, Caffrey M. Controlling release from the lipidic cubic phase. Amino acids, peptides, proteins and nucleic acids, Journal of Controlled Release, 107 (2005) 97-111. 